\title{
Cathelicidin induces epithelial-mesenchymal transition to promote airway remodeling in smoking-related chronic obstructive pulmonary disease
}

\author{
Zhiming Jiang ${ }^{1,2,3}$, Yuke Zhang ${ }^{3}$, Yibing Zhu ${ }^{4}$, Chong $\mathrm{Li}^{3}$, Lei Zhou ${ }^{3}$, Xiaolin Li $^{3}$, Fuxiang Zhang ${ }^{3}$, \\ Xianming Qiu ${ }^{3}$, Yiqing $\mathbf{Q u}{ }^{1}$ \\ ${ }^{1}$ Department of Pulmonary and Critical Care Medicine, Qilu Hospital, Cheeloo College of Medicine, Shandong University, Jinan, China; \\ ${ }^{2}$ Department of Pulmonary and Critical Care Medicine, Shandong Qianfoshan Hospital, Cheeloo College of Medicine, Shandong University, Jinan, \\ China; ${ }^{3}$ Department of Pulmonary and Critical Care Medicine, the First Affiliated Hospital of Shandong First Medical University, Jinan, China; \\ ${ }^{4}$ Medical Research and Biometrics Center, National Center of Cardiovascular Disease, Fuwai Hospital, Peking Union Medical College, Beijing, \\ China \\ Contributions: (I) Conception and design: Y Qu; (II) Administrative support: Y Qu; (III) Provision of study materials or patients: Z Jiang, Y Zhang, C \\ Li, L Zhou, X Qiu; (IV) Collection and assembly of data: Y Zhang, Y Zhu, X Li, F Zhang; (V) Data analysis and interpretation: Z Jiang, Y Zhu, Y \\ $\mathrm{Qu}$; (VI) Manuscript writing: All authors; (VII) Final approval of manuscript: All authors. \\ Correspondence to: Yiqing Qu, MD. Department of Pulmonary and Critical Care Medicine, Qilu Hospital, Cheeloo College of Medicine, Shandong \\ University, 107 Wenhuaxi Road, Jinan 250012, China. Email: quyiqing@sdu.edu.cn.
}

Background: Epithelial-mesenchymal transition (EMT) is an important characteristic in the remodeling of airways that occurs in chronic obstructive pulmonary disease (COPD). Cigarette smoke is a potential driving factor of this EMT in COPD. However, the mechanisms by which cigarette smoke induce EMT remain uncertain. Cathelicidin has been implicated as a causal factor of airway inflammation and mucus hypersecretion in smoking-related COPD. This study aimed to investigate whether cathelicidin induces EMT to promote airway remodeling in this disease.

Methods: Human lung tissue was collected from smokers with COPD and smokers without COPD. The EMT markers E-cadherin and vimentin were examined by immunohistochemistry. Mouse models of COPD were established by taking mice with airway cathelin-related antimicrobial peptide (CRAMP), the murine homologue of cathelicidin, either upregulated or downregulated by intranasal introduction of lentiviral vectors and then exposing them to cigarette smoke. E-cadherin and vimentin expression in the airways of the model mice was examined using immunofluorescence. Tumor necrosis factor alpha (TNF- $\alpha$ ) converting enzyme (TACE), transforming growth factor alpha (TGF- $\alpha$ ), and epidermal growth factor receptor (EGFR) expression was analyzed by Western blot. Additionally, NCI-H292 human airway epithelial cells, both with and without cathelicidin downregulation, were stimulated with cigarette smoke extract (CSE) and LL-37 synthetic peptide, a bioactive fragment of cathelicidin. This was done to confirm that the TACE/TGF- $\alpha$ / EGFR signaling pathway is activated in humans exposed to cigarette smoke.

Results: Significant EMT was found in the small airways of smokers both with and without COPD, as well as in the airways of COPD model mice. Downregulation of CRAMP in COPD mice, however, ameliorated airway EMT induced by cigarette smoke. Conversely, upregulation of CRAMP enhanced airway EMT in vivo; TACE, TGF- $\alpha$, and EGFR were found to be involved in this process. In vitro, EMT induced by CSE and LL-37 was inhibited by blocking TACE, TGF- $\alpha$, and EGFR expression.

Conclusions: Cathelicidin promotes airway EMT by activating the TACE/TGF- $\alpha$ /EGFR signaling pathway. This mediates smoking-induced airway remodeling in the pathogenesis of COPD.

Keywords: Chronic obstructive pulmonary disease (COPD); epithelial-mesenchymal transition (EMT); smoking; cathelicidin 
Submitted Mar 05, 2020. Accepted for publication Oct 23, 2020.

doi: 10.21037/atm-20-2196

View this article at: http://dx.doi.org/10.21037/atm-20-2196

\section{Introduction}

Chronic obstructive pulmonary disease (COPD) is a significant global health problem that has substantial social and economic impacts (1). COPD is currently the third leading cause of death worldwide (2). A large-scale survey taken in 2015 showed that the overall prevalence of COPD in China was as high as $13.6 \%$ (3). However, the mechanisms for the pathogenesis of COPD are still largely unknown, which makes preventing and treating this disease difficult. COPD is characterized by persistent airflow limitation and is associated with airway and lung tissue abnormalities caused by toxic particle and gas exposure (4). Cigarette smoke is considered the most important risk factor for COPD (4). The primary pathological features of COPD are inflammation and airway remodeling, emphysema, and airway mucus hypersecretion $(5,6)$. Airway remodeling, particularly that of the small airways (airways $<2 \mathrm{~mm}$ in internal diameter), is the primary cause of airflow limitation (6). Airway remodeling is a complicated process in which the fibrosis caused by epithelial-mesenchymal transition (EMT) may play an important role $(7,8)$.

EMT is an important biological phenomenon during which epithelial cells lose epithelial properties and assume the phenotype of stromal cells (9). During this process, the expression of E-cadherin, keratin, and other epithelial markers is decreased, while the expression of mesenchymal markers, such as vimentin, $\alpha$-smooth muscle actin, fibronectin, and N-cadherin, is elevated $(7,9)$. EMT has been shown to promote embryonic development, tissue repair, and fibrosis, as well as invasion and metastasis of a variety of tumors (9). For the past few decades, in vivo and in vitro studies have demonstrated that EMT is active in COPD and plays an important role in the remodeling of both large and small airways $(8,10-17)$. However, the driving factors and precise mechanisms of airway EMT have yet to be fully defined. Currently, cigarette smoke is believed to be a key risk factor for EMT in COPD. Studies have shown that cigarette smoke can stimulate airway epithelial cells to produce free radical species that can cause EMT, as well as stimulate these cells to upregulate urokinase type plasminogen activator receptor (uPAR), which promotes EMT $(13,18,19)$. However, cigarette smoke is a complex mixture of chemicals and compounds; the mechanisms by which cigarette smoke induces EMT are no doubt sophisticated.

Cathelicidin is an important part of the innate immune system in humans; the homologue in mice is known as cathelin-related antimicrobial peptide (CRAMP) (20). Cathelicidin has various biological functions, including anti-infection functions, and the regulation of inflammation and immunity, promotion of tissue repair, and regulation of tumor cell behavior (20). Studies have shown that cathelicidin is involved in the pathogenesis of COPD (21-26). Overexpression of cathelicidin in the airway epithelium promotes the development of smoking-related COPD by inducing airway inflammation, mucus hypersecretion, and fibroblast collagen production $(21,23)$. However, it is unclear whether cathelicidin induces airway remodeling by activating EMT in smoking-related COPD.

In this study, we investigated the expression of cathelicidin and EMT markers in the airway epithelium of smokers both with and without COPD, following which we established COPD mouse models to explore whether cigarette smoke can induce airway EMT in vivo. To investigate the role of cathelicidin in smoking-induced EMT, the expression of cathelicidin in the airway epithelium of the model mice was upregulated or downregulated by intranasal administration of lentiviral vectors. Finally, we explored in vitro the possible molecular mechanisms of cathelicidin-induced airway EMT by downregulating the expression of cathelicidin in NCI-H292 human airway epithelial cells.

\section{Methods}

\section{Subjects}

Three groups of subjects comprising 10 non-smokers without COPD (non-smokers group), 9 smokers without COPD (smokers group), and 9 smokers with COPD (COPD group), respectively, were recruited from Qilu Hospital (Cheeloo College of Medicine, Shandong University), where all subjects underwent therapeutic lung resection for the removal of tumors. Noncancerous lung 
tissue blocks $15-25 \mathrm{~mm}$ in size located at least $5 \mathrm{~cm}$ from the tumor margin were collected from resected specimens, fixed in $10 \%$ formaldehyde, and embedded in paraffin. According to the guidelines of the Global Initiative of Chronic Obstructive Lung Disease (GOLD), all subjects underwent spirometry and those with a ratio of forced expiratory volume in one second over forced vital capacity $\left(\mathrm{FEV}_{1} / \mathrm{FVC}\right)$ of less than $70 \%$ were diagnosed as having COPD. Patients were excluded from this study if they had respiratory infections, bronchial asthma, bronchiectasis, congestive heart failure, or neuromuscular disorders. This study was conducted in accordance with the Declaration of Helsinki (as revised in 2013) and was approved by the Ethics Committee of Qianfoshan Hospital of Shandong University (approval number 2019S044). Informed consent was obtained from all subjects.

\section{Histopathological analysis of lung tissue}

Human lung tissue taken from the subjects was cut into sections of $4-\mu \mathrm{m}$ in thickness, mounted on slides, and then stained using Masson's trichrome staining to assess airway remodeling. Cathelicidin, E-cadherin, and vimentin expression in the small airways of the subjects was determined by immunohistochemistry. The sections of lung tissue were stained with primary cathelicidin antibodies (1:50), E-cadherin antibodies (1:100), and vimentin antibodies $(1: 100)$ at the proper respective dilutions. Primary antibodies were purchased from Abcam, Cambridge, USA. Following incubation with horseradish peroxidase-conjugated secondary antibodies, the staining colors were developed with diaminobenzidine. The stained sections were assessed by a researcher blind to the subject groups. Three randomly selected fields in each section were evaluated at $200 \times$ magnification. The mean staining density was analyzed using Image-Pro Plus 6.0 software and shown as the average integrated optical density (IOD) per stained area $\left(\mu \mathrm{m}^{2}\right)$.

\section{Preparation of lentiviral vectors}

The murine CRAMP lentiviral vectors used in this study were developed by GeneChem (Shanghai, China). Briefly, CRAMP cDNA was amplified by polymerase chain reaction (PCR) and inserted into GV115 lentiviral vectors to generate GV/CRAMP under the control of a cytomegalovirus promoter. The insertion and orientation of CRAMP genes were confirmed by plasmid sequencing.
The construct was then co-transfected with a mixture of packaging plasmids (pHelper1.0 and pHelper2.0) into human embryonic kidney $293 \mathrm{~T}$ cells $\left(1.5 \times 10^{6}\right)$ in serumfree medium using Lipofectamine (Gibco, Carlsbad, USA). After $12 \mathrm{~h}$, the transfection medium was replaced with complete medium and cells were incubated for another $72 \mathrm{~h}$. Viral supernatant was then collected and filtered with a filter with $0.45-\mu \mathrm{m}$ pore size. $\mathrm{GV} / \beta$-galactosidase ( $\beta$-gal) was used as the positive control. Expression of $\beta$-gal was determined by $\mathrm{X}-\mathrm{Gal}$ staining with titers calculated by counting the foci of blue cells per well and dividing by the dilution factor. GV/CRAMP was then produced by transient transfection of $293 \mathrm{~T}$ cells. Viral stocks were stored at $-80{ }^{\circ} \mathrm{C}$. Lentiviral vectors containing CRAMP small interfering RNA (siRNA) (a sequence designed by Thermo: GCAGTCCCTAGACACCAAT) used in this study were constructed in a similar manner.

\section{Animals and treatment}

Thirty male BALB/c mice (6 weeks old) weighing 18-22 g were purchased from SPF Biotechnology (Beijing, China). The mice were housed in specific-pathogen-free conditions and acclimated for 7 days before experimentation began. The mice were randomly divided into 6 groups $(\mathrm{n}=5$ for each group): the control group, COPD group, COPD plus lentiviral negative control (COPD + NC) group, COPD plus CRAMP downregulation (COPD + CRAMP) group, CRAMP upregulation $\left(\mathrm{CRAMP}^{+}\right)$group, and COPD plus CRAMP upregulation (COPD + CRAMP $^{+}$) group. These mice were exposed to mainstream cigarette smoke from Kentucky 3R4F research cigarettes in a whole-body exposure system (Huironghe Technology, Beijing, China) at $200 \mathrm{mg}$ TPM $/ \mathrm{m}^{3}$ for $2 \mathrm{~h}$ per day, 5 days a week for 8 weeks. In accordance with a method previously reported (27), the mice in the CRAMP ${ }^{+}$COPD + NC, COPD + CRAMP ${ }^{+}$, and COPD + CRAMP ${ }^{-}$groups were administrated $10^{8}$ TU of corresponding lentiviral vectors intranasally every 10 days throughout the experimental period with the first instillation on day 1 and the last instillation on day 50 . Control group mice were kept in a similar environment without exposure to cigarette smoke or intranasal administration of lentiviral vectors. On completion of experimental protocol, the mice were sacrificed and their lungs were collected for microscopic and molecular analysis as described in previous publications (28). This animal experimentation was performed under a project license (approval number 2019S044) granted by the Ethics 
Committee of Qianfoshan Hospital of Shandong University in compliance with the Chinese National Guidelines for the Care and Use of Animals.

\section{Histopathological analysis of mouse lung tissue}

The lung tissue collected from the mice experimental groups was fixed, cut into 4- $\mu \mathrm{m}$ sections, and stained with Masson's trichrome staining to assess airway remodeling. Immunofluorescence assays were conducted as described in previous publications (22). Briefly, the slides were blocked with $5 \%$ bovine serum albumin (Sigma, Darmstadt, Germany) for $1 \mathrm{~h}$ at room temperature and then incubated with primary antibodies at $4{ }^{\circ} \mathrm{C}$ overnight. The following primary antibodies were used at the appropriate dilutions: anti-CRAMP (1:50), anti-E-cadherin (1:100), and antivimentin (1:100) (Abcam, Cambridge, USA). The slides were incubated the next day with fluorescence-conjugated secondary antibodies (Beyotime, Shanghai, China) for $1 \mathrm{~h}$ at room temperature. Then, the slides were examined and photographed using fluorescence microscopy (ECLIPSE C1, Nikon, Japan) with 40× magnification. The fluorescence intensities of CRAMP, E-cadherin, and vimentin were calculated using Image-Pro Plus 6.0 software and shown as IOD/area.

\section{Western blot assay}

The protein levels of tumor necrosis factor alpha (TNF- $\alpha$ ) converting enzyme (TACE), transforming growth factor alpha (TGF- $\alpha$ ), and epidermal growth factor receptor (EGFR) were detected by Western blot. Lung tissue lysates from the sacrificed mice were denatured and quantified with a bicinchoninic acid (BCA) (Beyotime, Shanghai, China) assay. Samples containing $20 \mu \mathrm{g}$ protein were separated using 10\% SDS-PAGE gel, transferred to polyvinylidene fluoride (PVDF) membranes, and probed with antibodies for TACE $(1: 1,000)$, TGF- $\alpha(1: 500)$ and EGFR $(1: 1,000)$ at appropriate dilutions. All antibodies were obtained from Abcam (Cambridge, USA). $\beta$-actin was used as the endogenous loading control.

\section{Preparation of cigarette smoke extract (CSE)}

CSE was prepared using a method described in a previous publication (22). Briefly, 3R4F cigarettes (University of Kentucky, USA) were conditioned at $22{ }^{\circ} \mathrm{C}$ and $60 \%$ relative humidity for $48 \mathrm{~h}$ before use, following which 5 cigarettes were combusted using an experimental apparatus driven by a peristaltic pump. The resultant smoke was bubbled through $10 \mathrm{ml}$ of phosphate buffered saline (PBS). The suspension was adjusted to $\mathrm{pH} 7.2$ and filtered through a $0.22-\mu \mathrm{m}$ pore size filter to remove bacteria. This solution was considered to be $100 \%$ CSE. The freshly prepared CSE solution was diluted with Roswell Park Memorial Institute (RPMI) 1640 medium immediately before use.

\section{Cell culture}

NCI-H292, a human airway epithelial cell line, was obtained from the Chinese National Infrastructure of Cell Line Resource. The cells were cultured in RPMI 1640 medium with fetal bovine serum (10\%), penicillin $(100 \mathrm{U} / \mathrm{mL})$, and streptomycin $(100 \mu \mathrm{g} / \mathrm{mL})$, and then supplemented at $37{ }^{\circ} \mathrm{C}$ in a humidified $5 \% \mathrm{CO}_{2} / 95 \%$ air water-jacketed incubator. Once the cells reached near $80 \%$ confluence, they were serum-starved for $24 \mathrm{~h}$ before further treatments were conducted. The in vitro experiments were performed using serum-free media.

\section{LL-37 siRNA transfection}

LL-37 siRNA (Santa Cruz Biotechnology, Germany) transfections were conducted according to protocols provided by Santa Cruz Biotechnology, Inc. Briefly, NCI-H292 cells were seeded in 6-well tissue culture plates and incubated in serum-free and antibiotic-free medium until near $80 \%$ confluency. Solution A was prepared with $6 \mu \mathrm{L}$ siRNA duplex added into $100 \mu \mathrm{L}$ siRNA transfection medium (Santa Cruz Biotechnology, Germany), and solution B was prepared with $6 \mu \mathrm{L}$ siRNA transfection reagent (Santa Cruz Biotechnology, Germany) into 100 $\mu \mathrm{L}$ siRNA transfection medium. Then $0.8 \mathrm{~mL}$ siRNA transfection medium was added to the siRNA transfection reagent mixture (Solution $\mathrm{A}+$ Solution $\mathrm{B}$ ), which was used to incubate with the washed cells for 24 hours in transfection. Control siRNA-A (Santa Cruz Biotechnology, Germany) was used as a negative control.

\section{Immunofuorescence of airway epithelial NCI-H292 cells}

NCI-H292 cells were seeded on chamber slides in serumfree medium. Once the cells reached near $80 \%$ confluence, they were incubated with CSE (5\%) or LL-37 synthetic peptide $(5 \mu \mathrm{g} / \mathrm{mL})$ with or without pretreatment with EGFR inhibitor AG1478, TGF- $\alpha$ neutralized antibodies, 
Table 1 Clinical characteristics of the subjects

\begin{tabular}{|c|c|c|c|}
\hline Characteristics & Non-smokers & Smokers & COPD patients \\
\hline Subjects (n) & 10 & 9 & 9 \\
\hline Age (years) & $53.1(7.1)$ & $63.4(10.3)^{\mathrm{a}}$ & $60.8(11.1)^{\mathrm{a}}$ \\
\hline $\begin{array}{l}\text { Gender (male/ } \\
\text { female) }\end{array}$ & $6 / 4$ & $9 / 0$ & $7 / 2$ \\
\hline $\begin{array}{l}\text { Smoking history } \\
\text { (pack-years) }\end{array}$ & 0 & $41.6(30.8)$ & $40.0(25.9)$ \\
\hline $\mathrm{FEV}_{1}(\%$ predicted $)$ & $99.7(13.6)$ & $112.71(16.7)$ & $81.3(17.6)^{a, b}$ \\
\hline $\mathrm{FEV}_{1} / \mathrm{FVC}(\%)$ & $78.1(4.8)$ & $75.8(3.2)$ & $61.5(10.66)^{a, b}$ \\
\hline
\end{tabular}

Data are expressed as mean (s.d.). ${ }^{a} \mathrm{P}<0.01$ vs. non-smokers; ${ }^{\mathrm{b}} \mathrm{P}<0.01$ vs. smokers. COPD, chronic obstructive pulmonary disease; FEV1, forced expiratory volume in one second; FVC, forced vital capacity.

and TACE inhibitor TAPI-1. The slides were then washed in PBS and fixed in 4\% paraformaldehyde for $20 \mathrm{~min}$. Next, the cells were permeabilized with Triton X100 (1\%) for $20 \mathrm{~min}$ and further incubated with corresponding primary antibodies overnight. The following antibodies were used at appropriate dilutions: anti-CRAMP (1:50), anti-E-cadherin (1:100), and anti-vimentin (1:100) (Abcam, Cambridge, USA). The slides were incubated with fluorescenceconjugated secondary antibodies (Beyotime, Shanghai, China) the next day for $1 \mathrm{~h}$ at room temperature. Images were taken using fluorescence microscopy (ECLIPSE C1, Nikon, Japan) with 200× magnification.

\section{Statistical analysis}

Prior to statistical analysis, the Gauss test was performed on the data. Nonparametric analyses were carried out on the appropriate data. The Kruskal-Wallis test and MannWhitney $\mathrm{U}$ test were used to make comparisons between patient groups. One-way analysis of variance (ANOVA) was used to compare in vivo data between multiple treatment groups. $\mathrm{P}<0.05$ was considered statistically significant. Statistical analyses were performed using SPSS 26.0 software (SPSS Inc., Chicago, IL, USA).

\section{Results}

\section{Clinical data}

The subjects' primary clinical data are shown in Table 1. No significant difference was observed in smoking history between smokers with COPD and smokers without COPD $(\mathrm{P}<0.05)$. The ratio of forced expiratory volume in one second over the predicted $\mathrm{FEV}_{1} \%$ value $\left(\mathrm{FEV}_{1} / \mathrm{FEV}_{1} \%\right.$ predicted), as well as $\mathrm{FEV}_{1} / \mathrm{FVC}$, were significantly lower in COPD patients compared with non-smokers and smokers without COPD $(\mathrm{P}<0.01)$.

\section{Human small airway remodeling}

The small airways in human lung tissue were examined using Masson's trichrome staining. Significant collagen depositions in the small airway wall were found in smokers both with and without COPD (Figure 1), indicating airway remodeling had occurred in both of these groups.

\section{Immunostaining of cathelicidin and EMT markers in small airway epithelium}

The expression levels of cathelicidin, E-cadherin, and vimentin were determined by immunostaining. As shown in Figure 2, compared with non-smokers, the expression of cathelicidin and vimentin in the small airway epithelium was markedly upregulated in the smokers group and COPD group $(\mathrm{P}<0.01)$, and the expression of $\mathrm{E}$-cadherin showed the opposite trend $(\mathrm{P}<0.01)$. No significant difference existed in cathelicidin, E-cadherin, or vimentin expression between smokers with COPD and smokers without COPD. These changes in EMT marker expression indicate that EMT had occurred in the small airways of smokers both with and without COPD. This is in accordance with the findings of previous studies $(12,15,16,29)$.

\section{Mouse small airway remodeling}

The small airways of different experimental groups of mice were examined using Masson's trichrome staining. Significant collagen deposition was observed in the airway walls of mice exposed to cigarette smoke and those with upregulated CRAMP, indicating that airway remodeling had occurred (Figure 3).

\section{CRAMP-induced airway EMT in a smoking-related COPD mouse model}

To investigate whether cathelicidin induces airway EMT in smoking-related COPD, we established a COPD animal model by exposing mice to cigarette smoke. Mouse models both with and without regulation of regional 

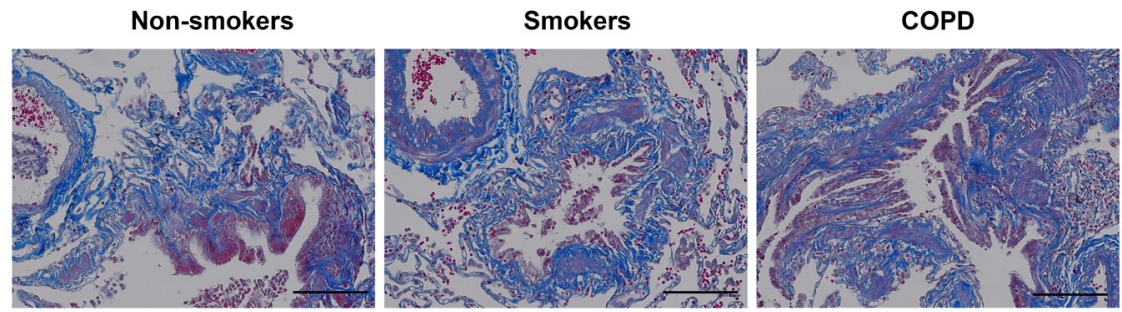

Figure 1 Pathological changes in the small airways of non-smokers, smokers without COPD, and COPD patients (Masson's trichome staining). All images are 200× magnification. Scale bars $=100 \mu \mathrm{m}$. COPD, chronic obstructive pulmonary disease.

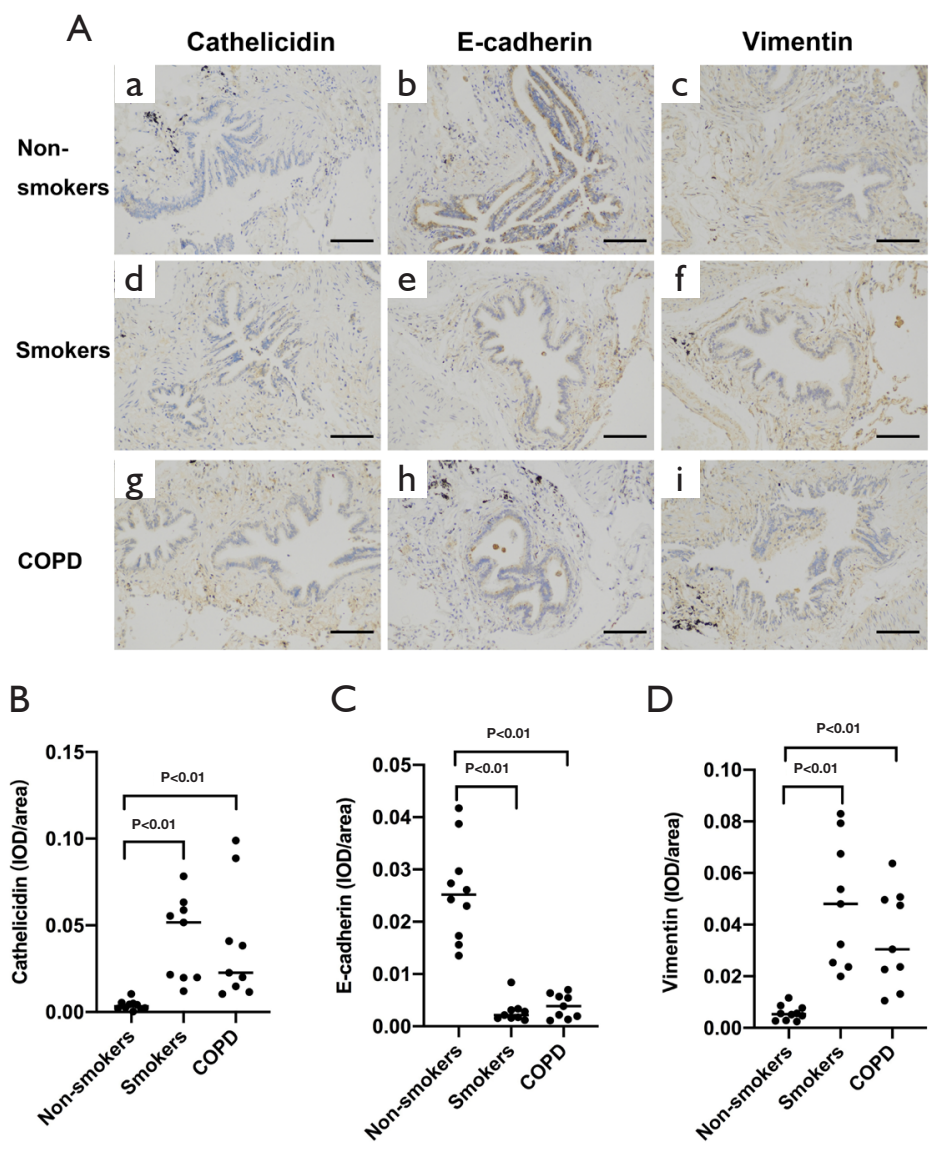

Figure 2 The expression of cathelicidin and EMT markers in the small airways of non-smokers, smokers without COPD, and COPD patients. (A) Immunostaining of cathelicidin, E-cadherin, and vimentin in (a-c) non-smokers, (d-f) smokers, and (g-i) COPD patients. All images are 200× magnification. Scale bars $=100 \mu \mathrm{m}$. Immunostaining density (IOD/area) of (B) cathelicidin, (C) E-cadherin, and (D) vimentin in the small airway epithelium of non-smokers, smokers, and COPD patients. The bars on the graphs indicate median IOD/area. EMT, epithelial-mesenchymal transition; COPD, chronic obstructive pulmonary disease.

CRAMP expression in the airway epithelium were used. Immunofluorescence was used to evaluate the expression levels of CRAMP and EMT markers. We found that, compared with the control group, the expression of
CRAMP and vimentin was significantly increased and the expression of E-cadherin was significantly decreased in the COPD group (Figure 4). These results suggest that cigarette smoking could induce cathelicidin overexpression 

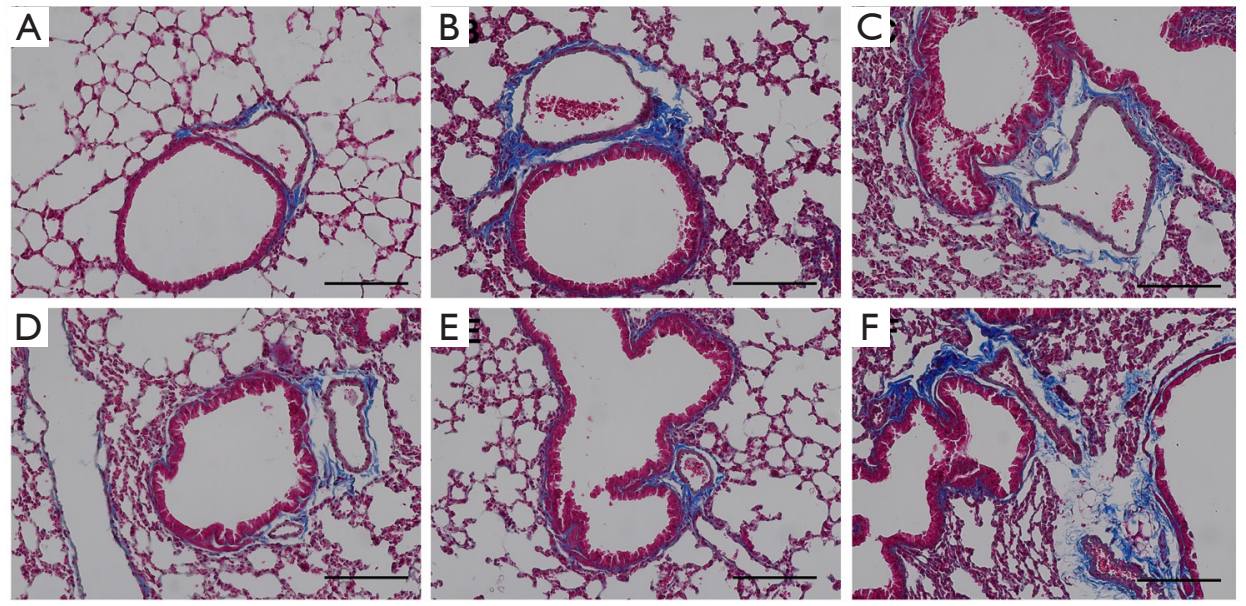

Figure 3 Pathological changes in the airways in mouse models (Masson's trichome staining). The images represent the airways of six experimental groups: the control group, COPD group, COPD plus lentiviral vector negative control (COPD + NC) group, COPD plus CRAMP downregulation (COPD + CRAMP-) group, CRAMP upregulation $\left(\mathrm{CRAMP}^{+}\right)$group, and COPD plus CRAMP upregulation $\left(\mathrm{COPD}+\mathrm{CRAMP}^{+}\right)$group. All images are $200 \times$ magnification. Scale bars $=100 \mu \mathrm{m}$. COPD, chronic obstructive pulmonary disease; CRAMP, cathelin-related antimicrobial peptide.

and airway EMT in the development of COPD. This is in accordance with our findings above concerning human specimens and those of previous studies $(13,22,29)$. We further found that upregulation of CRAMP in the airway epithelium can cause the expression of EMT markers to change in mice not exposed to cigarette smoke in a similar way to that observed in COPD group mice. This indicates that CRAMP has the potential to induce EMT (Figure 4). Additionally, when CRAMP was downregulated in the airway epithelium, smoking-induced EMT was significantly inhibited (Figure 4). These results suggest that CRAMP acts as a critical mediator between cigarette use and airway EMT in the pathogenesis of COPD.

\section{CRAMP-induced airway EMT via TACE/TGF- $\alpha / E G F R$ signaling patbway in vivo}

In the pathogenesis of COPD, both cathelicidin and cigarette smoke can activate airway epithelial cells to promote the release of inflammatory mediators and mucins via the TACE/TGF- $\alpha$ /EGFR signaling pathway $(24,30,31)$. To determine whether CRAMP induces EMT through the TACE/TGF- $\alpha$ /EGFR signaling pathway, we used western blot assays to examine the expression of TACE, TGF- $\alpha$, and EGFR in lung tissue taken from mouse models. Our results showed that, compared with the control group, the expression of TACE, TGF- $\alpha$, and EGFR at the protein level was significantly increased in the COPD group and the $\mathrm{CRAMP}^{+}$group (Figure 5). This indicates that both cigarette smoke and CRAMP can activate the TACE/ TGF- $\alpha$ /EGFR signaling pathway in vivo. When CRAMP was downregulated, the expression of TACE, TGF- $\alpha$, and EGFR induced by cigarette smoke (COPD + CRAMP-group) was significantly lower than that of the COPD group (Figure 5). This suggests that CRAMP activation of the TACE/TGF- $\alpha /$ EGFR signaling pathway plays an important role in mediating smoking-induced airway EMT in the development of COPD.

\section{Cathelicidin mediation of CSE-induced EMT in vitro}

To investigate the effect of cigarette smoke on EMT in vitro, we treated human airway epithelial NCI-H292 cells, some transfected with LL-37 siRNA and some not, with 5\% CSE. Firstly, we examined the expression of cathelicidin using immunofluorescence and found that CSE can induce cathelicidin expression in NCI-H292 cells (Figure 6). Additionally, when the NCI-H292 cells were transfected with LL-37 siRNA, cathelicidin induction by CSE was inhibited (Figure 6). This indicates that the LL-37 siRNA transfection effectively inhibited cathelicidin induction. Secondly, we examined the expression of the EMT markers 


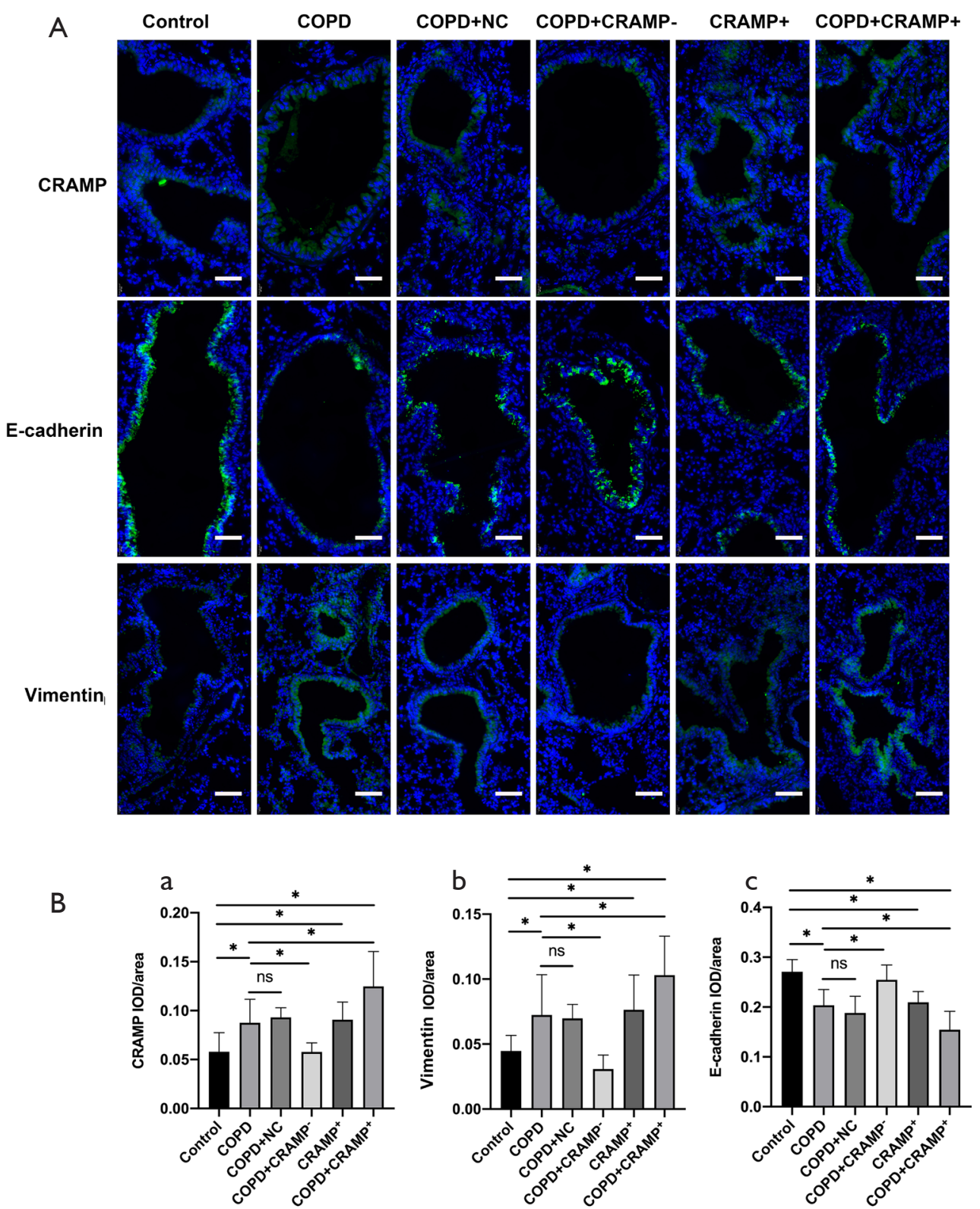

Figure 4 The expression of CRAMP and EMT markers in the airways in mouse models. (A) Immunofluorescence images of CRAMP, E-cadherin, and vimentin in the airways of six mouse model groups: the control group, COPD group, COPD plus lentiviral vector negative control (COPD + NC) group, COPD plus CRAMP downregulation (COPD + CRAMP $\left.{ }^{-}\right)$group, CRAMP upregulation (CRAMP+) group, and COPD plus CRAMP upregulation $\left(\mathrm{COPD}+\mathrm{CRAMP}^{+}\right.$) group. Scale bars $=30 \mu \mathrm{m}$. (B) Comparison of fluorescence intensity (IOD/ area) of (a) CRAMP, (b) E-cadherin, and (c) vimentin between the experimental groups. Data are presented as mean $\pm \mathrm{SD}$; ${ }^{*} \mathrm{P}<0.01$; ns $=$ no significance. CRAMP, cathelin-related antimicrobial peptide; EMT, epithelial-mesenchymal transition; COPD, chronic obstructive pulmonary disease. 

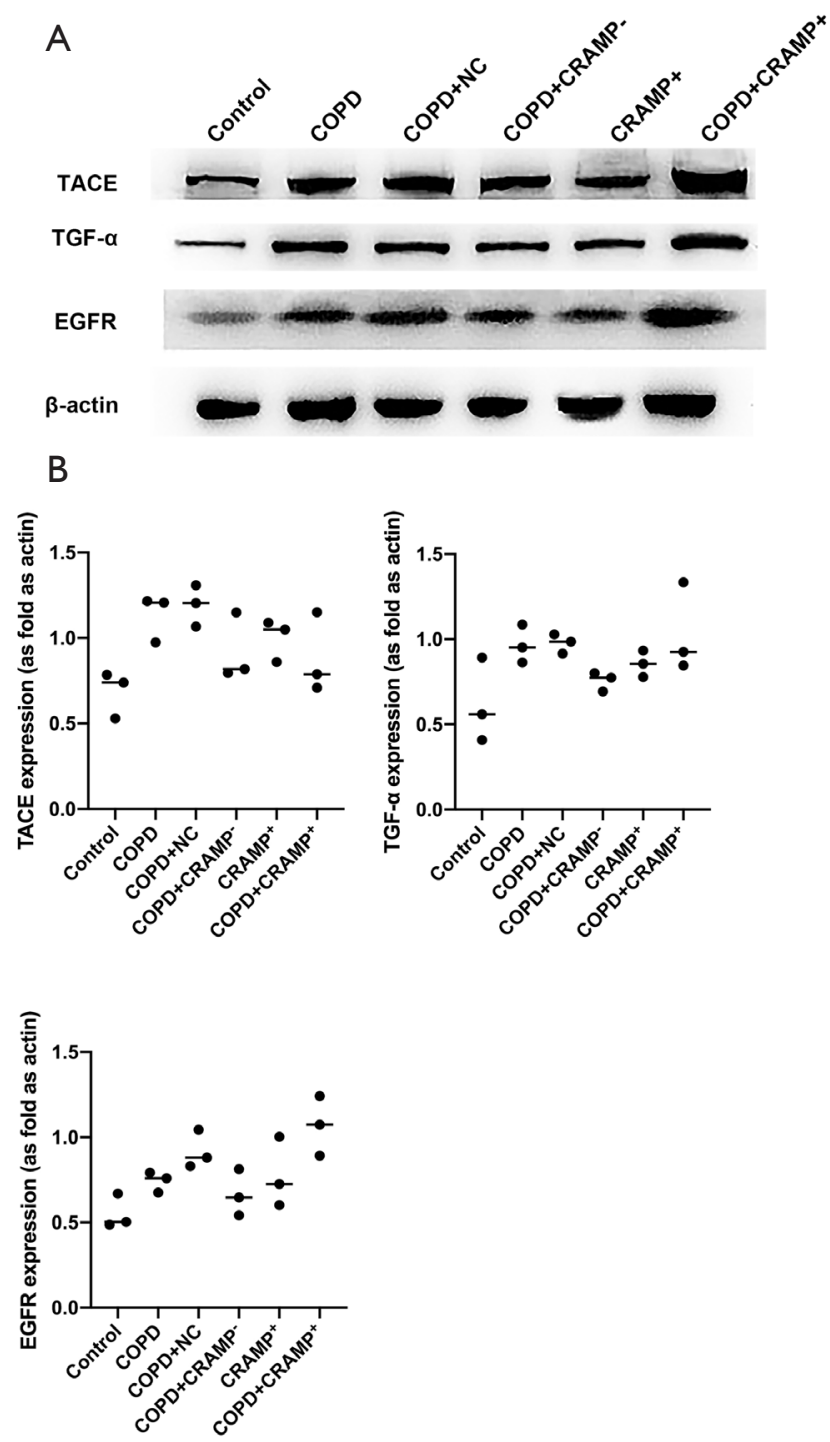

Figure 5 Changes in TACE, TGF- $\alpha$, and EGFR protein expression in the lung tissue of different mouse model experimental groups as shown by western blot. (A) Representative images of protein expression. The six groups were the control group, COPD group, COPD plus lentiviral vector negative control $(\mathrm{COPD}+\mathrm{NC})$ group, COPD plus CRAMP downregulation $\left(\mathrm{COPD}+\mathrm{CRAMP}^{-}\right)$group, $\mathrm{CRAMP}$ upregulation $\left(\mathrm{CRAMP}^{+}\right)$group, and COPD plus CRAMP upregulation (COPD + CRAMP ${ }^{+}$group. (B) Analysis of protein expression levels. The bars on the graphs indicate median expression levels. TACE, TNF- $\alpha$ converting enzyme; TGF- $\alpha$, transforming growth factor alpha; EGFR, epidermal growth factor receptor; COPD, chronic obstructive pulmonary disease; CRAMP, cathelin-related antimicrobial peptide.

E-cadherin and vimentin using immunofluorescence and found that E-cadherin expression was inhibited by CSE, while vimentin expression was increased (Figure 7). This suggests that CSE can induce airway EMT in vitro. However, EMT induction by CSE was inhibited when the cells were pretreated with LL-37 siRNA (Figure 7), indicating that cathelicidin mediated the process of CSE- induced EMT.

CSE-induced EMT mediated via the cathelicidinstimulated TACE/TGF-a/EGFR patbway

To further validate the potential molecular mechanism of airway EMT induced by cigarette smoke, NCI-H292 cells 


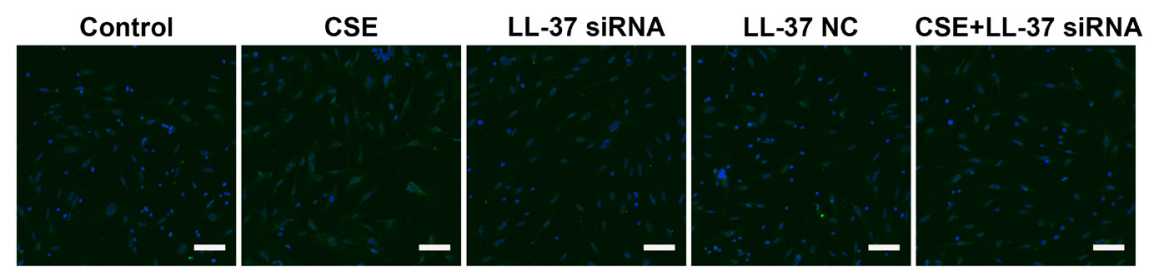

Figure 6 The expression of cathelicidin induced by CSE in NCI-H292 cells. Before treatment with 5\% CSE, the NCI-H292 cells were pretreated with LL-37 siRNA to downregulate cathelicidin expression. After $24 \mathrm{~h}$ of treatment, cathelicidin expression was examined by immunofluorescence. Scale bars $=100 \mu \mathrm{m}$. CSE, cigarette smoke extract; siRNA, small interfering RNA.

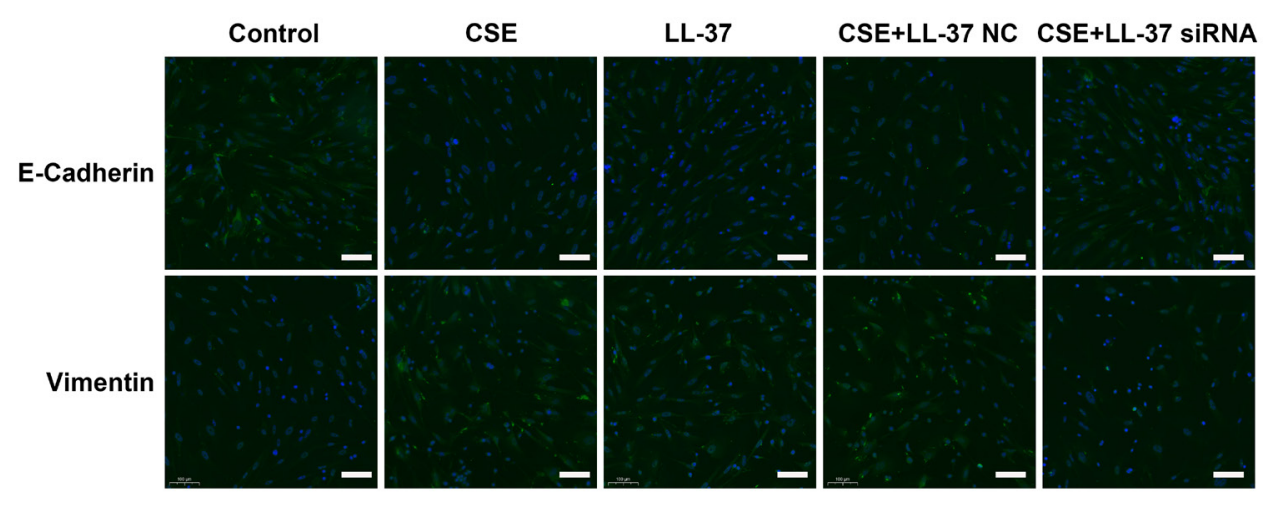

Figure 7 The expression of EMT markers E-cadherin and vimentin induced by CSE and LL-37 in NCI-H292 cells. The cells were treated with $5 \%$ CSE and LL-37 synthetic peptide $(5 \mu \mathrm{g} / \mathrm{mL})$ for $24 \mathrm{~h}$ after treatment with LL-37 siRNA to downregulate cathelicidin expression. The expression of E-cadherin and vimentin was then examined by immunofluorescence. Scale bars $=100 \mu \mathrm{m}$. EMT, epithelial-mesenchymal transition; CSE, cigarette smoke extract; siRNA, small interfering RNA.

were pretreated with EGFR inhibitor AG1478, TGF- $\alpha$ neutralizing antibody, and TACE inhibitor TAPI-1 to block the effects of EGFR, TGF- $\alpha$, and TACE, respectively. Then, the cells were treated with $5 \mu \mathrm{g} / \mathrm{mL}$ LL-37 synthetic peptide, and the expression of the EMT markers E-cadherin and vimentin was examined by immunofluorescence. We found that the expression of E-cadherin was inhibited and the expression of vimentin was increased by exogeneous LL-37 peptide (Figure 8), indicating that cathelicidin is capable of inducing EMT. On the other hand, these changes were attenuated by the pretreatment with AG1478, TGF- $\alpha$ neutralizing antibody and TAPI-1, suggesting that cathelicidin-induced EMT can be suppressed by inhibitors of EGFR, TGF- $\alpha$ and TACE in vitro. All these results indicate that cathelicidin stimulation of the TACE/TGF- $\alpha$ / EGFR signaling pathway is responsible for CSE-induced EMT in airway epithelial cells.

\section{Discussion}

In this study, we showed that cathelicidin and the EMT phenomenon coexisted in the airways of smokers with and without COPD. Further, we showed that cigarette smoke can induce the expression of CRAMP in the airway epithelium of mice, with CRAMP expression promoting EMT via the TACE/TGF- $\alpha$ /EGFR signaling pathway, causing airway remodeling in the development of COPD.

Previous studies have shown that EMT is involved in airway remodeling in COPD (8). This remodeling occurs primarily in the small airways, where EMT disturbs the epithelium by increasing subepithelial mesenchymal cells and thickening airway walls, which causes the airways to narrow $(8,32)$. As cigarette smoke is considered to be the most important determinant of COPD, it is highly likely to affect airway EMT $(13,29)$. Researchers have explored the effects of cigarette smoke on airway EMT, both in 


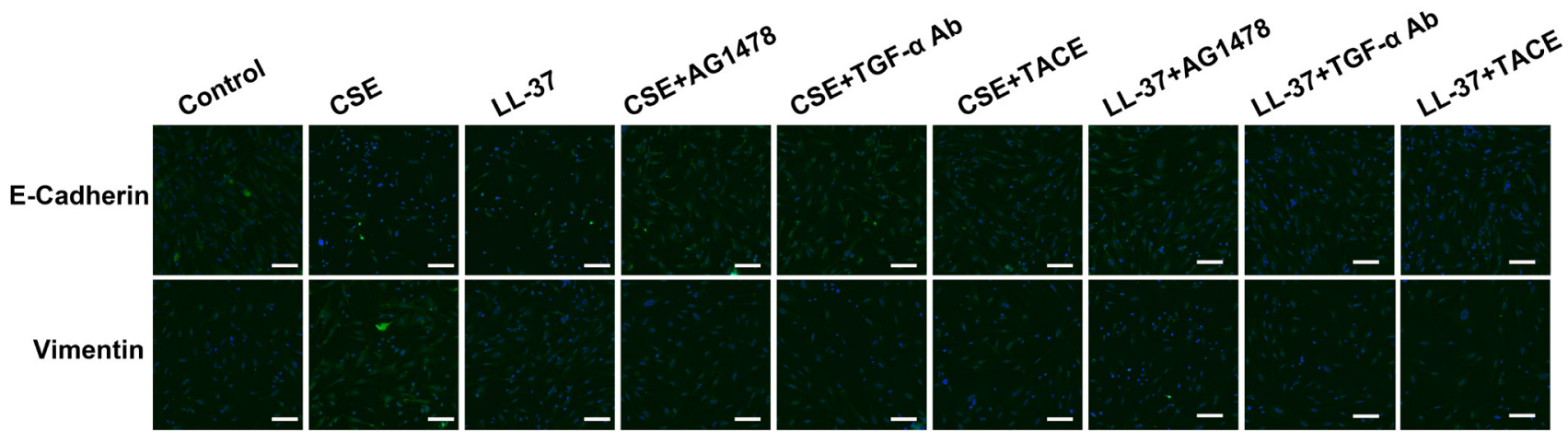

Figure 8 The TACE/TGF- $\alpha$ /EGFR signaling pathway was involved in cathelicidin-induced EMT. The CSE and LL-37-induced expression of EMT markers E-cadherin and vimentin in NCI-H292 cells were examined by immunofluorescence following treatment with the EGFR blocker AG1478 (2 $\mu \mathrm{mol} / \mathrm{L})$, TACE inhibitor TAPI-1 $(10 \mu \mathrm{mol} / \mathrm{L})$, and TGF- $\alpha$ neutralizing antibody $(1 \mu \mathrm{g} / \mathrm{mL})$ for $30 \mathrm{~min}$. Scale bars $=100 \mu \mathrm{m}$. TACE, TNF- $\alpha$ converting enzyme; TGF- $\alpha$, transforming growth factor alpha; EGFR, epidermal growth factor receptor; EMT, epithelial-mesenchymal transition; CSE, cigarette smoke extract.

vivo and in vitro $(13,15,17)$. In line with these studies, our results showed that EMT occurred in the small airways of smokers both with and without COPD and that exposure to cigarette smoke can induce airway EMT in mice. This further validates the causal relationship between cigarette smoke and EMT, although the precise mechanisms by which cigarette smoke causes EMT remain unclear. As cigarette smoke consists of a wide variety of chemicals and compounds, the proposed mechanisms of smoking-induced EMT are complicated. Studies have shown that various types of biomolecules, such as TROP2 (33), IL-17A (34), GDF15 (34), cullin4A (35), ROS (18), and uPAR (19,29) are implicated in this process and affect EMT via multiple signaling pathways, including TGF- $\beta 1 /$ Smad/ERK (36), TGF- $\beta 1 / \mathrm{Akt} / \mathrm{Smad} / \mathrm{mTOR}$ (37), Akt/P 38 MAPK, $\mathrm{Akt} / \mathrm{PKB}$ (38), and EGFR/Akt/ $\beta$-catenin (39). In the present study, we found for the first time that the TACE/TGF- $\alpha /$ EGFR signaling pathway mediated smoking-induced airway EMT in COPD when activated by cathelicidin.

The TACE/TGF- $\alpha$ /EGFR signaling pathway can be activated in the airway epithelium by various factors and plays an important role in the pathogenesis of COPD $(24,30,40)$. Various in vitro studies have shown that cigarette smoke (30), Pseudomonas aeruginosa, and lipopolysaccharides (LPS) (40), as well as cathelicidin (24), can activate human airway epithelial cells via the TACE/TGF- $\alpha$ /EGFR signaling pathway, resulting in the overexpression of mucin; this promotes airway mucus hypersecretion in COPD. Our results demonstrated that the expression of TACE, TGF- $\alpha$, and EGFR in airway tissue was upregulated in COPD mouse models exposed to smoke, which suggests that the TACE/TGF- $\alpha / E G F R$ signaling pathway is activated by cigarette smoke in vivo. Previous studies have also shown that the TACE/TGF- $\alpha / E G F R$ signaling pathway is responsible for EMT, although these results are primarily related to epithelial tumors. For example, EMT induced by activation of the TACE/TGF- $\alpha$ /EGFR signaling pathway was shown to promote tumor cell invasion and metastasis in prostate and liver cancer $(41,42)$. Both previous studies and our results suggest that activation of the TACE/TGF- $\alpha$ / EGFR signaling pathway might mediate smoking-induced EMT in the pathogenesis of COPD.

Cathelicidin is an important part of the human innate immune system that can be expressed constitutively or inductively in the airway epithelium (20). Cathelicidin has various biological functions, including anti-infection functions and regulating inflammation, immunity, and tumor behavior, as well as promoting the pathogenesis of COPD (20). As cathelicidin functions by activating the TACE/TGF- $\alpha$ /EGFR signaling pathway in smokingrelated airway mucus hypersecretion in COPD (24), we further explored whether cathelicidin played a role in smoking-related EMT, another key pathological characteristic of COPD. Our results demonstrated that the expression of cathelicidin in the airway epithelium of smokers both with and without COPD was elevated, and exposure to cigarette smoke can upregulate CRAMP expression in the airway epithelium of mice. This suggests that cigarette smoke influences cathelicidin expression in the airways, which is supported by previous studies 
$(22,23)$. When CRAMP expression was downregulated in the airways, cigarette smoke-induced expression of TACE, TGF- $\alpha$, and EGFR was significantly decreased when EMT was inhibited, and vice versa. These in vivo results suggest that the TACE/TGF- $\alpha$ /EGFR signaling pathway might be involved in cathelicidin-induced EMT. To further validate the molecular mechanism of smoking-associated EMT, we conducted in vitro experiments using human airway epithelial cells and found that CSE could induce EMT, and this CSE-induced EMT was inhibited when cathelicidin was downregulated in cells. Additionally, we found that TACE, TGF- $\alpha$, and EGFR inhibitors could block EMT induced by exogeneous LL-37 synthetic peptide. These in vivo and in vitro results suggest that cathelicidin-induced activation of the TACE/TGF- $\alpha$ /EGFR pathway fulfils an important bridging role between cigarette smoke and airway EMT in the development of COPD.

Notably, our results showed no significant difference in cathelicidin and EMT marker expression between smokers with COPD and those without. This finding does not align with those of previous studies $(22,29)$. This may be attributed to the fact that, in the present study, smokers with COPD and smokers without COPD had similar histories of heavy smoking. Additionally, other factors that influence EMT in COPD patients, such as regional and systemic inflammation $(17,43)$, might be obscured by the influence of smoking. Another possibility is that the sample size of this study was relatively small, which might have affected our results. Future studies should recruit more participants, to clarify possible differences between smokers with COPD and smokers without COPD.

In addition to these, there were some other limitations to this study. Firstly, due to the fact that more males smoke than females, we recruited primarily male subjects. The role of EMT in the pathogenesis of COPD may differ according to sex. Secondly, we measured EMT using semiquantitative immunostaining. However, immunostaining is only sufficient to demonstrate the presence or absence of EMT and cannot measure the degree to which this phenomenon has occurred. Finally, we used NCI-H292 cells to investigate the molecular mechanisms of EMT. It is important to note that this is an immortalized epitheliumderived cell line, and some differences might exist between this cell line and human airway epithelial cells in nature. To further validate the role of cathelicidin-induced EMT in the pathogenesis of smoking-related COPD, future studies should be large scale and quantitative, and also recruit more female subjects and use primary human airway epithelial cells in their experiments.

\section{Conclusions}

In conclusion, this study demonstrated that cathelicidin was correlated with EMT in the small airways of smokers both with and without COPD. Cathelicidin was also shown to promote airway EMT by activating the TACE/TGF- $\alpha$ / EGFR signaling pathway to mediate smoking-induced airway remodeling in the pathogenesis of COPD.

\section{Acknowledgments}

We thank AME Editing Services (AME Publishing Company) for editing the English draft of our manuscript.

Funding: The research was supported by grants from the Science and Technology Foundation of Shandong Province (2007GG3002008, 2015GGH318002, and 2015GSF118064), the Shandong Provincial Natural Science Foundation (ZR2015CL012), the Medical Science and Technology Development Program of Shandong Province (2017WS363 and 2017WS761), and the Qianyipeiyu Program (QYPY2019NSFC0605).

\section{Footnote}

Data Sharing Statement: Available at http://dx.doi. org/10.21037/atm-20-2196

Peer Review File: Available at http://dx.doi.org/10.21037/ atm-20-2196

Conflicts of Interest: All authors have completed the ICMJE uniform disclosure form (available at http://dx.doi. org/10.21037/atm-20-2196). The authors have no conflicts of interest to declare.

Ethical Statement: The authors are accountable for all aspects of the work in ensuring that questions related to the accuracy or integrity of any part of the work are appropriately investigated and resolved. Participants gave written informed consent before the study, and the study protocol was approved by the Ethics Committee of Qianfoshan Hospital of Shandong University (approval number 2019S044) in accordance with the Declaration of Helsinki (as revised in 2013). All efforts were made to minimize animal suffering, and the animal experiments were performed under a project license (approval number 
2019S044) granted by the Ethics Committee of Qianfoshan Hospital of Shandong University in compliance with the Chinese National Guidelines for the Care and Use of Animals.

Open Access Statement: This is an Open Access article distributed in accordance with the Creative Commons Attribution-NonCommercial-NoDerivs 4.0 International License (CC BY-NC-ND 4.0), which permits the noncommercial replication and distribution of the article with the strict proviso that no changes or edits are made and the original work is properly cited (including links to both the formal publication through the relevant DOI and the license). See: https://creativecommons.org/licenses/by-nc-nd/4.0/.

\section{References}

1. Mirza S, Clay RD, Koslow MA, et al. COPD Guidelines: A Review of the 2018 GOLD Report. Mayo Clin Proc 2018;93:1488-502.

2. Adeloye D, Chua S, Lee C, et al. Global Health Epidemiology Reference G. Global and regional estimates of COPD prevalence: Systematic review and meta-analysis. J Glob Health 2015;5:020415.

3. Sun Y, Sin DD. Crisis of COPD in China: the future is now. Lancet Respir Med 2018;6:404-5.

4. (GOLD) GIfCOlD. 2019 Global strategy for prevention, diagnosis and management fo COPD. [cited]. Available online: https://goldcopd.org/wp-content/ uploads/2018/11/GOLD-2019-v1.7-FINAL-14Nov2018WMS.pdf

5. Hogg JC, Chu F, Utokaparch S, et al. The nature of smallairway obstruction in chronic obstructive pulmonary disease. N Engl J Med 2004;350:2645-53.

6. Hogg JC, Pare PD, Hackett TL. The Contribution of Small Airway Obstruction to the Pathogenesis of Chronic Obstructive Pulmonary Disease. Physiol Rev 2017;97:529-52.

7. Jolly MK, Ward C, Eapen MS, et al. Epithelialmesenchymal transition, a spectrum of states: Role in lung development, homeostasis, and disease. Dev Dyn 2018;247:346-58.

8. Courtney JM, Spafford PL. The Role of EpithelialMesenchymal Transition in Chronic Obstructive Pulmonary Disease. Cells Tissues Organs 2017;203:99-104.

9. Nieto MA, Huang RY, Jackson RA, et al. Emt: 2016. Cell 2016;166:21-45

10. Ward C, Forrest IA, Murphy DM, et al. Phenotype of airway epithelial cells suggests epithelial to mesenchymal cell transition in clinically stable lung transplant recipients. Thorax 2005;60:865-71.

11. Willis BC, Liebler JM, Luby-Phelps K, et al. Induction of epithelial-mesenchymal transition in alveolar epithelial cells by transforming growth factor-beta1: potential role in idiopathic pulmonary fibrosis. Am J Pathol 2005;166:1321-32.

12. Molloy EL, Adams A, Moore JB, et al. BMP4 induces an epithelial-mesenchymal transition-like response in adult airway epithelial cells. Growth Factors 2008;26:12-22.

13. Milara J, Peiro T, Serrano A, et al. Epithelial to mesenchymal transition is increased in patients with COPD and induced by cigarette smoke. Thorax 2013;68:410-20.

14. Kamitani S, Yamauchi Y, Kawasaki S, et al. Simultaneous stimulation with TGF-beta1 and TNF-alpha induces epithelial mesenchymal transition in bronchial epithelial cells. Int Arch Allergy Immunol 2011;155:119-28.

15. Sohal SS, Reid D, Soltani A, et al. Reticular basement membrane fragmentation and potential epithelial mesenchymal transition is exaggerated in the airways of smokers with chronic obstructive pulmonary disease. Respirology 2010;15:930-8.

16. Mahmood MQ, Sohal SS, Shukla SD, et al. Epithelial mesenchymal transition in smokers: large versus small airways and relation to airflow obstruction. Int J Chron Obstruct Pulmon Dis 2015;10:1515-24.

17. Sohal SS. Epithelial and endothelial cell plasticity in chronic obstructive pulmonary disease (COPD). Respir Investig 2017;55:104-13.

18. Gorowiec MR, Borthwick LA, Parker SM, et al. Free radical generation induces epithelial-to-mesenchymal transition in lung epithelium via a TGF-beta1-dependent mechanism. Free Radic Biol Med 2012;52:1024-32.

19. Wang Q, Wang Y, Zhang Y, et al. The role of uPAR in epithelial-mesenchymal transition in small airway epithelium of patients with chronic obstructive pulmonary disease. Respir Res 2013;14:67.

20. van Harten RM, van Woudenbergh E, van Dijk A, et al. Cathelicidins: Immunomodulatory Antimicrobials. Vaccines (Basel) 2018;6:63.

21. Jiang YY, Xiao W, Zhu MX, et al. The effect of human antibacterial peptide LL-37 in the pathogenesis of chronic obstructive pulmonary disease. Respir Med 2012;106:1680-9.

22. Sun C, Zhu M, Yang Z, et al. LL-37 secreted by epithelium promotes fibroblast collagen production: a potential 
mechanism of small airway remodeling in chronic obstructive pulmonary disease. Lab Invest 2014;94:991-1002.

23. Zhang Y, Jiang Y, Sun C, et al. The human cathelicidin LL-37 enhances airway mucus production in chronic obstructive pulmonary disease. Biochem Biophys Res Commun 2014;443:103-9.

24. Zhang Y, Zhu M, Yang Z, et al. The human Cathelicidin LL-37 induces MUC5AC mucin production by airway epithelial cells via TACE-TGF-alpha-EGFR pathway. Exp Lung Res 2014;40:333-42.

25. Golec M, Reichel C, Lemieszek M, et al. Cathelicidin LL37 in bronchoalveolar lavage and epithelial lining fluids from COPD patients and healthy individuals. J Biol Regul Homeost Agents 2012;26:617-25.

26. Golec M, Reichel C, Mackiewicz B, et al. Cathelicidin LL37, granzymes, TGF-beta 1 and cytokines levels in induced sputum from farmers with and without COPD. Ann Agric Environ Med 2009;16:289-97.

27. DuPage M, Dooley AL, Jacks T. Conditional mouse lung cancer models using adenoviral or lentiviral delivery of Cre recombinase. Nat Protoc 2009;4:1064-72.

28. Hautamaki RD, Kobayashi DK, Senior RM, et al. Requirement for macrophage elastase for cigarette smokeinduced emphysema in mice. Science 1997;277:2002-4.

29. Wang Q, Wang Y, Zhang Y, et al. Involvement of urokinase in cigarette smoke extract-induced epithelialmesenchymal transition in human small airway epithelial cells. Lab Invest 2015;95:469-79.

30. Shao MX, Nakanaga T, Nadel JA. Cigarette smoke induces MUC5AC mucin overproduction via tumor necrosis factor-alpha-converting enzyme in human airway epithelial (NCI-H292) cells. Am J Physiol Lung Cell Mol Physiol 2004;287:L420-7.

31. Tjabringa GS, Aarbiou J, Ninaber DK, et al. The antimicrobial peptide LL-37 activates innate immunity at the airway epithelial surface by transactivation of the epidermal growth factor receptor. J Immunol 2003;171:6690-6.

32. Rabe KF, Watz H. Chronic obstructive pulmonary disease. Lancet 2017;389:1931-40.

33. Liu Q, Li H, Wang Q, et al. Increased expression of TROP2 in airway basal cells potentially contributes to airway remodeling in chronic obstructive pulmonary disease. Respir Res 2016;17:159.

34. Jiang G, Liu CT, Zhang WD. IL-17A and GDF15 are able to induce epithelial-mesenchymal transition of lung epithelial cells in response to cigarette smoke. Exp Ther
Med 2018;16:12-20.

35. Ren Y, Zhang Y, Fan L, et al. The cullin4A is up-regulated in chronic obstructive pulmonary disease patient and contributes to epithelial-mesenchymal transition in small airway epithelium. Respir Res 2019;20:84.

36. Guan S, Xu W, Han F, et al. Ginsenoside Rg1 Attenuates Cigarette Smoke-Induced Pulmonary Epithelial-

Mesenchymal Transition via Inhibition of the TGF-beta1/ Smad Pathway. Biomed Res Int 2017;2017:7171404.

37. Luo Y, Ren Z, Du B, et al. Structure Identification of ViceninII Extracted from Dendrobium officinale and the Reversal of TGF-beta1-Induced Epithelial(-)Mesenchymal Transition in Lung Adenocarcinoma Cells through TGF-beta/Smad and PI3K/Akt/mTOR Signaling Pathways. Molecules 2019;24:144.

38. Jiang B, Guan $\mathrm{Y}$, Shen $\mathrm{HJ}$, et al. Akt/PKB signaling regulates cigarette smoke-induced pulmonary epithelialmesenchymal transition. Lung Cancer 2018;122:44-53.

39. Agraval H, Yadav UCS. MMP-2 and MMP-9 mediate cigarette smoke extract-induced epithelial-mesenchymal transition in airway epithelial cells via EGFR/Akt/ GSK3beta/beta-catenin pathway: Amelioration by fisetin. Chem Biol Interact 2019;314:108846.

40. Shao MX, Ueki IF, Nadel JA. Tumor necrosis factor alphaconverting enzyme mediates MUC5AC mucin expression in cultured human airway epithelial cells. Proc Natl Acad Sci U S A 2003;100:11618-23.

41. Caja L, Ortiz C, Bertran E, et al. Differential intracellular signalling induced by TGF-beta in rat adult hepatocytes and hepatoma cells: implications in liver carcinogenesis. Cell Signal 2007;19:683-94.

42. Tang J, Xiao L, Cui R, et al. CX3CL1 increases invasiveness and metastasis by promoting epithelial-tomesenchymal transition through the TACE/TGF-alpha/ EGFR pathway in hypoxic androgen-independent prostate cancer cells. Oncol Rep 2016;35:1153-62.

43. Câmara J, Jarai G. Epithelial-mesenchymal transition in primary human bronchial epithelial cells is Smaddependent and enhanced by fibronectin and TNF-alpha. Fibrogenesis Tissue Repair 2010;3:2.

Cite this article as: Jiang Z, Zhang Y, Zhu Y, Li C, Zhou L, Li X, Zhang F, Qiu X, Qu Y. Cathelicidin induces epithelialmesenchymal transition to promote airway remodeling in smoking-related chronic obstructive pulmonary disease. Ann Transl Med 2021;9(3):223. doi: 10.21037/atm-20-2196 